\title{
Angioma cavernoso cerebral gigante en paciente menor de un año: descripción de
}

\section{un caso}

\author{
H. Prinzo***; F. Martínez*,** y S. Carminatti*
}

*Servicio de Neurocirugía Pediátrica. Hospital Pereira Rossell (Ministerio de Salud Pública). Montevideo. Uruguay. **Servicio de Neurocirugía. Hospital de Clínicas (Universidad de la República). Montevideo. Uruguay.

\section{Resumen}

Los angiomas cavernosos son poco frecuentes en niños, y son menos frecuentes aún los angiomas cavernosos gigantes.

Describimos el caso clínico de un niño de 11 meses que consultó por un cuadro de crisis epilépticas de 5 meses de evolución. Los estudios de imagen (IRM) evidenciaron un angioma cavernoso gigante parietal derecho.

Se realizó una resección total de la lesión con control de las crisis.

Se reporta el caso dado lo excepcional de la lesión descrita en un paciente menor de un año.

PALABRAS CLAVE: Angioma cavernoso. Epilepsia secundaria. Cirugía de la epilepsia. Neurocirugía pediátrica.

Giant cerebral cavernous malformation in a child less than 1 year old: case report

\section{Summary}

Cerebral cavernous malformations are rarely described in children. Giant cavernous malformations are exceptional in this group of patients.

The authors reports a case of an eleven months boy, who begins at the sixth month of life with epilepsy. Medical therapy was advised and imagenological studies were carried out.

An IRM was performed, and shows a giant cavernous malformation in the right parietal lobe.

Surgery was carried out, with complete resection of the lesion. In the postoperative period and at more than a year of follow up, he was free of crisis.

We report the case because of the rarity of a giant cavernous angioma in a patient less than a year old.

Recibido: 15-11-07. Aceptado: 10-06-08
KEY WORDS: Cavernous angioma. Secondary epilepsy. Epilepsy surgery. Pediatric Neurosurgery.

\section{Introducción}

Los angiomas cavernosos o cavernomas, son lesiones relativamente poco frecuentes en la edad pediátrica. Pueden presentarse bajo múltiples formas clínicas o ser asintomáticos. De aquéllos que son sintomáticos, la mayoría se presentan con crisis ${ }^{1,4,8}$. El diagnóstico de estas lesiones ha aumentado en las últimas dos décadas por la accesibilidad cada vez mayor a estudios de imagen, en particular la Imagen por Resonancia Magnética (IRM).

En el presente reporte, los autores presentan de forma breve, un caso muy poco frecuente: un angioma cavernoso gigante en un niño menor de un año.

\section{Caso clínico}

D.A., 11 meses de edad. Procedente de medio rural. Embarazo controlado y sin alteraciones. Sin antecedentes familiares conocidos a destacar.

Comienza a los 6 meses de vida con crisis, caracterizadas por detención de la mirada y movimientos de chupeteo. Estas crisis eran esporádicas y comenzaron luego a hacerse más frecuentes por lo que consulta al pediatra que comienza tratamiento con fenobarbital. A los 10 meses de edad agrega crisis parciales de hemicuerpo izquierdo, con predominio en miembro superior. Por este motivo ingresa en el Centro Hospitalario Pereira Rossell, donde se hace tomografía de cráneo (TC) y luego resonancia nuclear magnética (RNM). No contamos con la TC inicial, pero la RNM evidenció una imagen de intensidad inhomogénea localizada en el lóbulo parietal derecho. En la citada imagen, se identificaban áreas hiper e hipointensas en $\mathrm{T} 1$, rodeado de una pequeña zona hipointensa. Dentro de la imagen descrita, se apreciaban áreas de vacío de flujo.

La lesión fue inicialmente interpretada como un tumor, sin descartar el angioma cavernoso.

Se realizó una cirugía planificada en base a los estudios imagenológicos. Se hizo un colgajo óseo libre exponiendo 

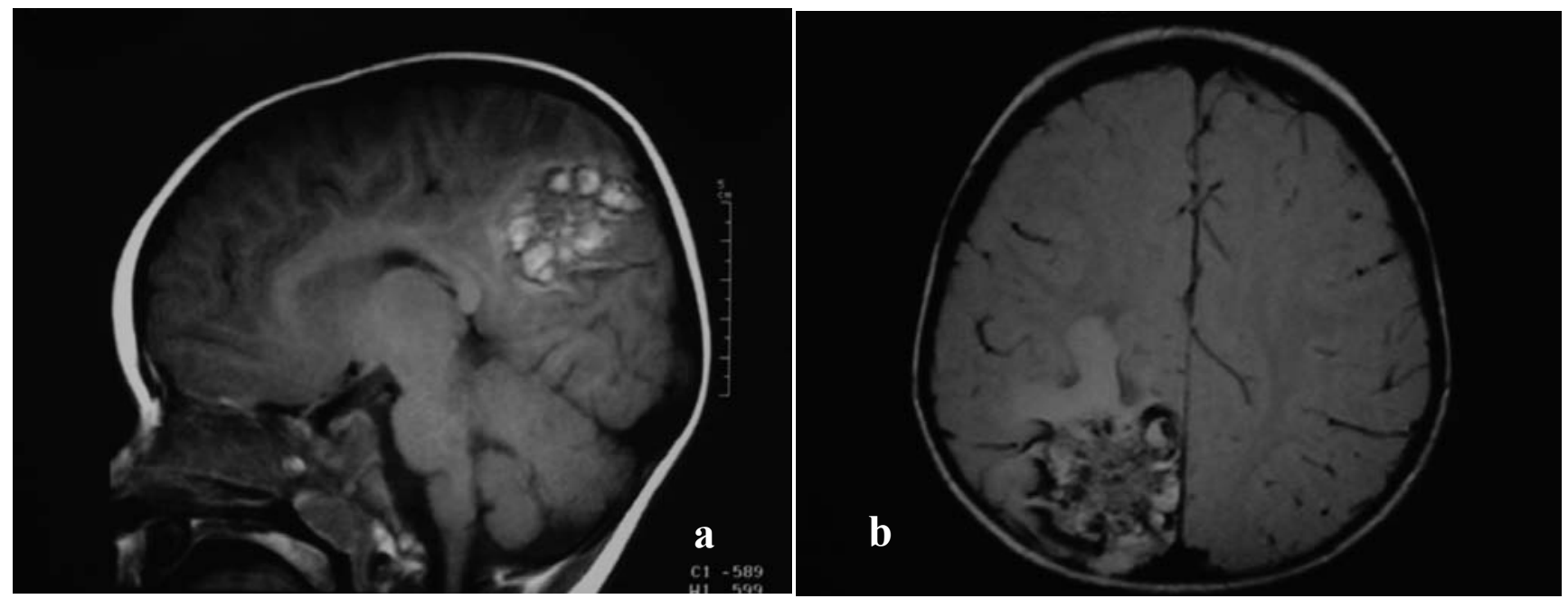

Figura 1. Imagen por IRM, secuencia T1.En las imágenes sagital y horizontal (a y b) se evidencia el angioma cavernoso, con áreas hipo e hiperintensas y un halo perilesional hipodenso.
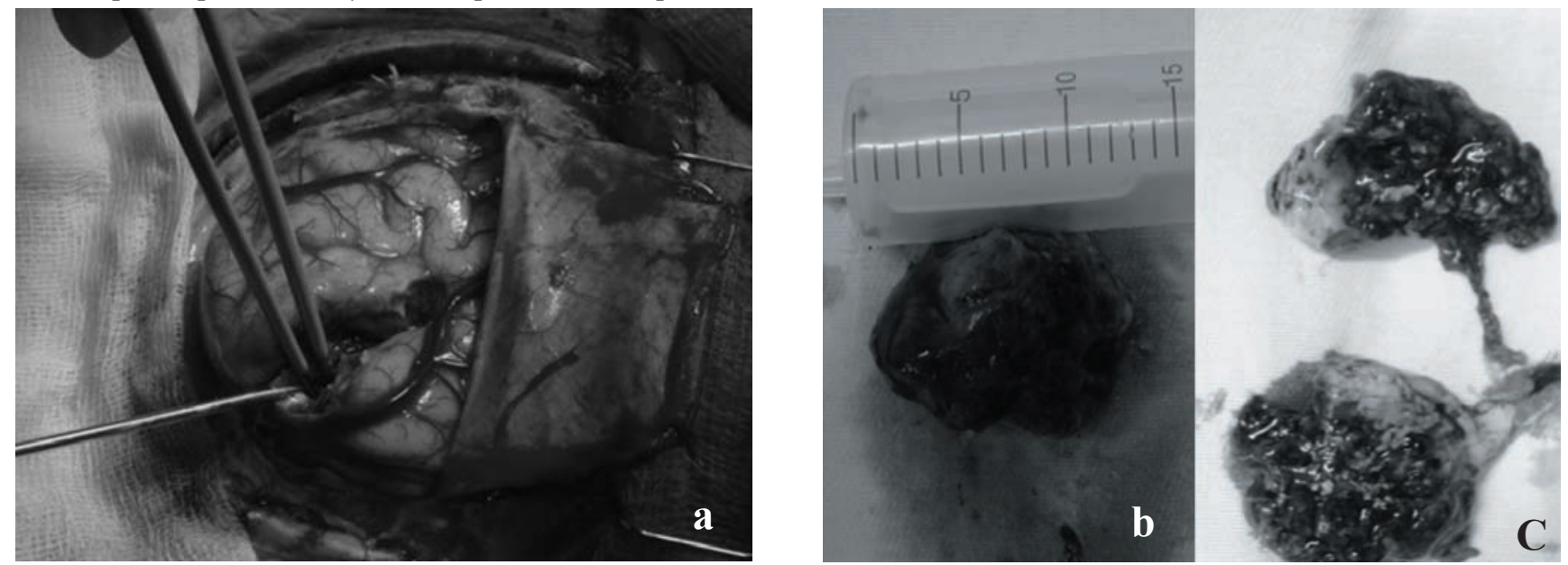

Figura 2. Resección quirúrgica del angioma cavernoso. En (a) se muestra la vena usada como referencia para la apertura del surco. Al hallar la lesión se labró un plano avascular alrededor de la misma (b) hasta hacer su exéresis completa (c).

el seno longitudinal superior y luego de la apertura dural se identificó una gruesa vena cortical, evidenciable en los estudios de imagen. Se realizó apertura del surco más cercano a la misma y se resecó en bloque una lesión voluminosa, con aspecto de angioma cavernoso. Este planteo fue corroborado por anatomía patológica. La evolución del niño fue excelente, sin agregar déficits neurológicos y fue dado de alta asintomático. El control de las crisis fue excelente sin presentarlas hasta el último control, un año después de la cirugía.

\section{Discusión}

Los angiomas cavernosos son lesiones vasculares relativamente poco frecuentes. La presencia de angiomas cavernosos en niños es aún menos frecuente, representando 1 ó $2 \%$ del total de los $\operatorname{casos}^{2,7}$ y en menores de un año son excepcionales. Por ejemplo, sobre 118 angiomas cavernosos tratados por $\mathrm{D}^{\prime}$ Angelo y cols. ${ }^{4}$, ningún paciente tenía menos de 19 meses de vida. Según Ng y cols. ${ }^{6}$, hasta el año 2006 solo se habían reportado 39 casos de angiomas cavernosos en menores de un año.

Por otro lado, los angiomas cavernosos gigantes (mayores a $6 \mathrm{cms}$.) son también excepcionales ${ }^{9}$. En la serie de $\mathrm{D}^{\prime}$ Angelo y cols. ${ }^{4}$, las medidas de los angiomas cavernosos resecados fueron entre 4 y $60 \mathrm{~mm}$, con una media de $26 \mathrm{~mm}$. Es la combinación de un angioma cavernoso gigante en un niño menor de un año, lo que motiva nuestro reporte.

Los angiomas cavernosos representan uno de los 4 grupos de malformaciones vasculares del sistema nervioso de la clasificación de McCormick, junto con las malformaciones arteriovenosas, teleangiectasias capilares $\mathrm{y}$ angiomas venosos (denominados actualmente malforma- 
ciones del desarrollo venoso) $)^{3,4}$.

Desde el punto de vista anatomopatológico, se trata de canales vasculares sinusoidales, de paredes delgadas, sin laminina, ni elastina y con pocas o ninguna fibras musculares $^{4}$.

Clínicamente pueden expresarse por epilepsia (cerca de $60 \%$ de los casos), sangrados intracraneanos, efecto de masa (por aumento de volumen de la lesión) o síntomas menos específicos, como cefaleas. Muchas veces son descubiertos de forma incidental y al momento del diagnóstico, entre 10 y $50 \%$ de los individuos presentan angiomas cavernosos múltiples $^{4,8}$. Para Cavalheiro y Menezes Braga ${ }^{3}$, las formas múltiples son menos frecuentes en niños.

En nuestro caso, la forma de presentación fueron las crisis que se iniciaron cerca del sexto mes de vida, aumentando su incidencia y cambiando su forma clínica cerca del décimo mes. Es probable que este cambio haya sido secundario a algún evento vascular dentro del propio angioma (sangrado) ya que el mismo presentaba diferentes intensidades en su interior, que evidencian hemosiderina en diferentes etapas evolutivas ${ }^{2,5,8}$.

$\mathrm{El}$ aspecto típico de los angiomas cavernosos en RNM es el de lesiones con aspecto de "granos de sal y pimienta" por las áreas de hiper e hipointensidad, rodeadas por un borde hipointenso de gliosis perilesional con depósitos de ferritina y hemosiderina ${ }^{2,5}$. En el interior de la lesión, las diferentes intensidades son dadas por: canales vasculares intralesionales, deoxihemoglobina y calcificaciones (hipointensos), vasos trombosados y metahemoglobina intra y extracelular (hiperintensidad). El halo perilesional hipointenso se debe al deposito de hemosiderina ${ }^{2,4,8}$. Van Lindert y cols. ${ }^{9}$ destacan que los angiomas gigantes pueden tener una presentación imagenológica diferente y que por esta causa y por su tamaño, no siempre se plantea este diagnóstico de inicio.

La topografía de la lesión, si bien dista de ser excepcional, también es poco frecuente, dado que los angiomas cavernosos predominan francamente en los lóbulos frontal y temporal ${ }^{8}$.

En nuestro caso, la resección del angioma sin la resección de la gnosis perilesional permitió el control total de las crisis, lo que también es encontrado por otros autores ${ }^{1,4}$.

\section{Conclusiones}

Se presenta un caso poco frecuente de un niño de 11 meses portador de un angioma gigante que se presentó clínicamente como una epilepsia secundaria a los 6 meses de vida. Se hizo resección completa de la lesión con remisión de las crisis.

Creemos de interés hacer este breve reporte por lo poco frecuente de la lesión aquí presentada.

\section{Bibliografía}

1. Awad, I., Jabbour, P.: Cerebral cavernous malformations and epilepsy. Neurosurg Focus 2006; 21 (1): E7.

2. Castillo, M.: Neurorradiología. Ediciones Journal, Buenos Aires. 2004.

3. Cavalheiro, S., Menezes Braga, F.: Cavernous hemangiomas. En: Choux, M., Di Rocco, C., Hockley, A., Walker, M. (eds): Pediatric Neurosurgery. Churchill Livingstone, Hong Kong, 1999: 691-702.

4. D’Angelo, V.A., De Bonis, C., Amoroso, R., Cali, A., D'Agruma, L., Guarnieri, V., Muscarella, L.A., Zelante, L., Bisceglia, M., Scarabino, T., Catapano, D.: Supratentorial cerebral cavernous malformations; clinical, surgical, and genetic involvement. Neurosurg Focus 2006; 21 (1): E9

5. Kuzniecky, R.I., Jackson, G.D.: Frontal lobe epilepsy. En: Kuzniecky, R.I., Jackson, G.D. (eds.): Magnetic resonance in epilepsy. Raven Press, New York, 1995: 183-202.

6. Ng, B.H., Mulyadi, E., Pereira, J.K., Ghedia, S., Pinner, J., Mowat, D., Vonau, M.: Familial cerebral cavernous haemangioma diagnosed in an infant with a rapidly growing cerebral lesion. Australas Radiol 2006; 50: 683-590 (Abstract).

7. Poch, J.M., Nogués, P.: Cavernomas. En: Villarejo, F., Martínez-Lage, J.F. (eds.): Neurocirugía Pediátrica. Ergon, Madrid, 2001: 442-445.

8. Rivera, P.P., Willinsky, R.A., Porter, P.J.: Intracranial cavernous malformations. Neuroimag Clin NA 2003; 13: 27 40.

9. van Lindert, E.J., Tan, T.C., Grotenhuis, J.A., Wesseling, P.: Giant cavernous hemangiomas: report of three cases. Neurosurg Rev 2007; 30: 83-92.

Prinzo, H.; Martínez, F.; Carminatti, S.: Angioma cavernoso cerebral gigante en paciente menor de un año: descripción de un caso. Neurocirugía 2009; 20: 54-56.

Correspondencia postal: Dr. Fernando Martínez. Mississipi 1536 Block D, Apartamento 501. Barrio Malvín, Montevideo, Uruguay. 